\title{
Blood Bank Services at Tertiary Care Center
}

\author{
Shubhangi Narayan Jibhkate (Bawankule)*, Neha Ramesh Patil, \\ Mukund YadavraoDhabale and Priyanka RamavatarAsawa
}

Department of Pathology, Indira Gandhi Government Medical College \& Hospital, Nagpur, Maharashtra. India

\begin{abstract}
Background: Audit is one mechanism for evaluating the effectiveness of the total quality system. Transfusion medicine or blood bank audit embraces all procedures from blood procurement to the long term consequences of transfusion.

Aims and Objectives: To understand the problems arising in day to day working of blood bank and to ensure the appropriate indication for issuing blood and blood components to different departments.

Materials and methods: Present audit of blood bank is retrospective study done for a year in tertiary care center in central region of India. Data collection of donors and whole blood issue is done for one year from 1st January 2016 to 31 st December 2016. Component separation had started from August 2016, so audit of component separation and its usage has been done for one year from 1stAugust 2016 to 31 stJuly 2017.

Results: In year 2016, total blood units collected were 6131 and issued were 5897. Majority of blood issue was to surgical wards 3790 compared to non-surgical wards 2107. Out of 6109, 203 were positive for transfusion transmitted infections (TTI). 20 transfusion reactions were reported in 2016. From 1st August 2016 to 31st July 2017, total 1060 Packed Red Cells (PRC) were prepared and 1009 were utilized, 1120 units of FFP were prepared, out of which 778 were utilized and 817 platelet concentrates were prepared but only 205 is utilized.
\end{abstract}

Conclusion: Results obtained from these types of retrospective studies will provide opportunity for finding strategies in improving the blood bank services.

\section{Keywords: Audit, Transfusion Services, Components.}

\section{Introduction}

The aim of blood transfusion services (BTS) should be to provide effective blood and blood products, which are as adequate as possible and safe. A Blood Transfusion Service is a complex organization, requiring careful designing and management. ${ }^{[1]}$ Availability of safe blood remains a major challenge for many developing countries. ${ }^{[2]}$ Audit is one mechanism for evaluating the effectiveness of the total quality system $\cdot{ }^{[3]}$ Comprehensive audits should be conducted periodically in accordance with written procedures and should consist of a review of a statistically significant number of records. ${ }^{[3]} \mathrm{An}$ audit tells whether the laboratory is performing its procedures, activities and policies correctly on time. ${ }^{[4]}$ It is an important public-health responsibility of national and state government to ensure a safe supply of blood and blood products and its appropriate and rational clinical use of blood. ${ }^{[5]}$ Transfusion medicine or blood bank audit embraces all procedures from blood procurement to the long term consequences of transfusion. ${ }^{[6]}$ Blood-bank activities include recruitment and retention of donors; collection, testing, processing, and storage of blood; and clinical use of blood and blood components and training. ${ }^{[7]}$
Specific parts or components of the blood are isolated, stored under conditions optimum for that component, and transfused to patients who need that specific component. ${ }^{[8]}$ Ordering clinician should consider the indication for appropriate transfusion of blood component and whole blood, so that indiscriminate use of blood is avoided.

\section{Aims And Objectives The main objectives were}

(1) To understand the usage of blood and blood components by different surgical and non-surgical departments and its appropriate indication for transfusion.

(2) To enlist problems arising in day to day working of blood bank and implementing solution for the same. The study was done to ensure optimum working of blood bank services.

\section{Materials and Methods}

Present audit of blood bank is done for a year in tertiary care center in central region of India. It is a retrospective study and the information of donors and recipients is taken from the records which are maintained by blood bank staff. Data collection for donors and whole blood issue is done for one year from $1^{\text {st }}$ January 2016 to $31^{\text {st }}$ December 2016. 
In August 2016 blood bank got permission for processing of components by Food and Drug Administration(FDA), so audit of component separation and its usage has been done for one year from $1^{\text {st }}$ August 2016 to $31^{\text {st }}$ July 2017.

Retrospective data is collected in blood bank which is licensed by FDA. Periodical inspection by FDA is needed to maintain the quality of blood transfusion services as well as for renewal of license. License is renewed after every five years by the FDA.

\section{Results}

As blood bank in present study is tertiary center, requirement of blood units is high and it is increasing every year. Average daily collection is nearly $15-17$ in number, average daily blood issue is nearly 18-22 (both whole blood \& component), average daily issue during night hours is 9-10 and average number of daily blood grouping done in blood bank is 47-50.

The collection of blood bank is constantly increasing. Total number of collection over the last five years is mentioned in figure 1. In 2016 total blood units collected were 6131 by voluntary donations in blood bank \&camps and as replacement for patient. In 2017 till October collection was 5507.

In year 2016, total blood units collected were 6131. Out of which 5619 (91\%) units were collected by voluntary collection and $512(9 \%)$ were collected as replacement for blood products given to the patients. In all donors, 5832(95\%) were male donors and 299(5\%) were female donors. Number of camps conducted over the last five years are mentioned in table number 1.Total number of blood issued in 2016 were 5897 . Out of which majority of blood issue was to surgical wards 3790 (64\%) compared to non-surgical wards 2107 (36\%). Out of 6109 collection, 203 $(3.3 \%)$ were positive of transfusion transmitted infections (TTI)in 2016. 118(1.9\%) were positive for Hepatitis B, $42(0.6 \%)$ for Human Immunodeficiency Virus (HIV), $29(0.4 \%)$ for Hepatitis C, $13(0.2 \%)$ for malaria parasite and $1(0.01 \%)$ for syphilis. 351 units were discarded in 2016 due to TTI positivity and less quantity in bags. In 2016, total transfusion reactions reported were 20. The most common clinical symptoms were fever, vomiting, itching with rash.

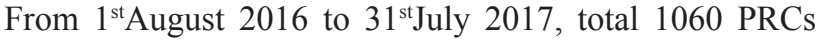
were prepared and 1009 were utilized , 1120 units of FFP were prepared, out of which 778 were utilized and 817 platelet concentrates were prepared but only 205 is utilized.

FDA has given minimum standards for manpower of category A blood bank in blood bank for annual collection of 1000-5000 according to this we have deficiency of 1 technician, 2 lab assistant, 2 clerk and 5 lab attendant and now our blood bank has entered category B having collection of 5000-10,000, we need more manpower for efficient working of blood bank.

\section{Discussion}

Audits identify areas of problems in transfusion services which can be corrected by education of doctors, formulation of practice guidelines and algorithms. ${ }^{[9]}$ In Maharashtra, all blood-banks and blood-storage centers have to maintain a record on blood collection and distribution. All regional blood-banks supplying blood units to blood-storage centers record the information on the number of blood units supplied, blood group of the supplied blood units, and dates of issue and expiry. ${ }^{[5]}$

An audit is performed to understand the established transfusion practice. After implementing the guidelines, a repeat audit needs to be done to guarantee they are being followed. This practice can also identify areas that need continued improvement. ${ }^{[10]}$ There should be communication between consultant and blood bank officer regarding appropriate component transfusion to ensure the optimum usage of component and proper follow up should be kept to decide the further guidelines. But overpopulated country like India its bit difficult to follow all the guidelines, as doctor to general population ratio is less in many regions of India. Thus, it seems clear that audit of transfusion can provide grounds for improvement not only in transfusion medicine, but also in other areas of medical practice. ${ }^{[6]}$

Our blood bank is FDA accredited. Laboratory accreditation is a procedure by which an authoritative body gives formal recognition that a laboratory is competent to carry out specific tasks based on third party assessment.

Table 1- Camps in last 4 years and present year (till September).

\begin{tabular}{|c|c|}
\hline Year & Total Camps \\
\hline 2013 & 71 \\
\hline 2014 & 77 \\
\hline 2015 & 79 \\
\hline 2016 & 101 \\
\hline 2017 (till October) & 105 \\
\hline
\end{tabular}




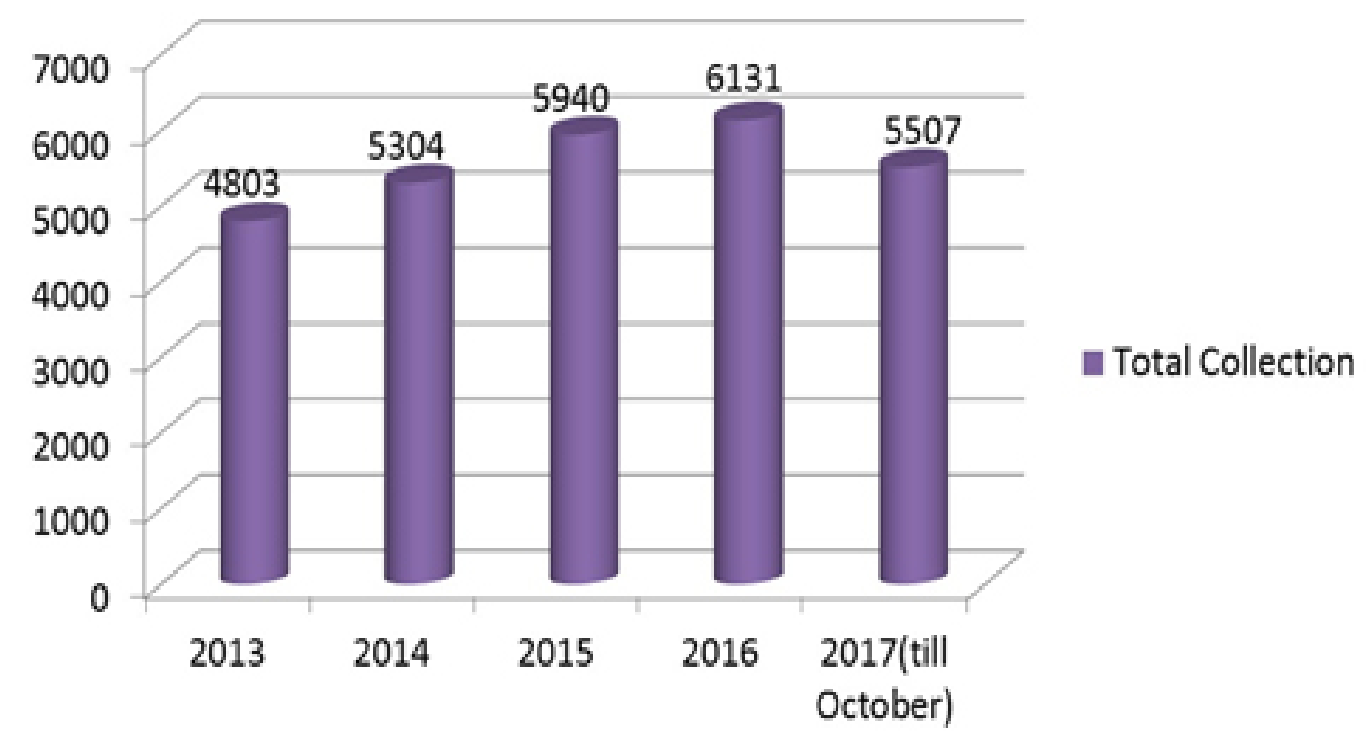

Fig. 1: Total Collection in last 4 years and present year(2017 till October).

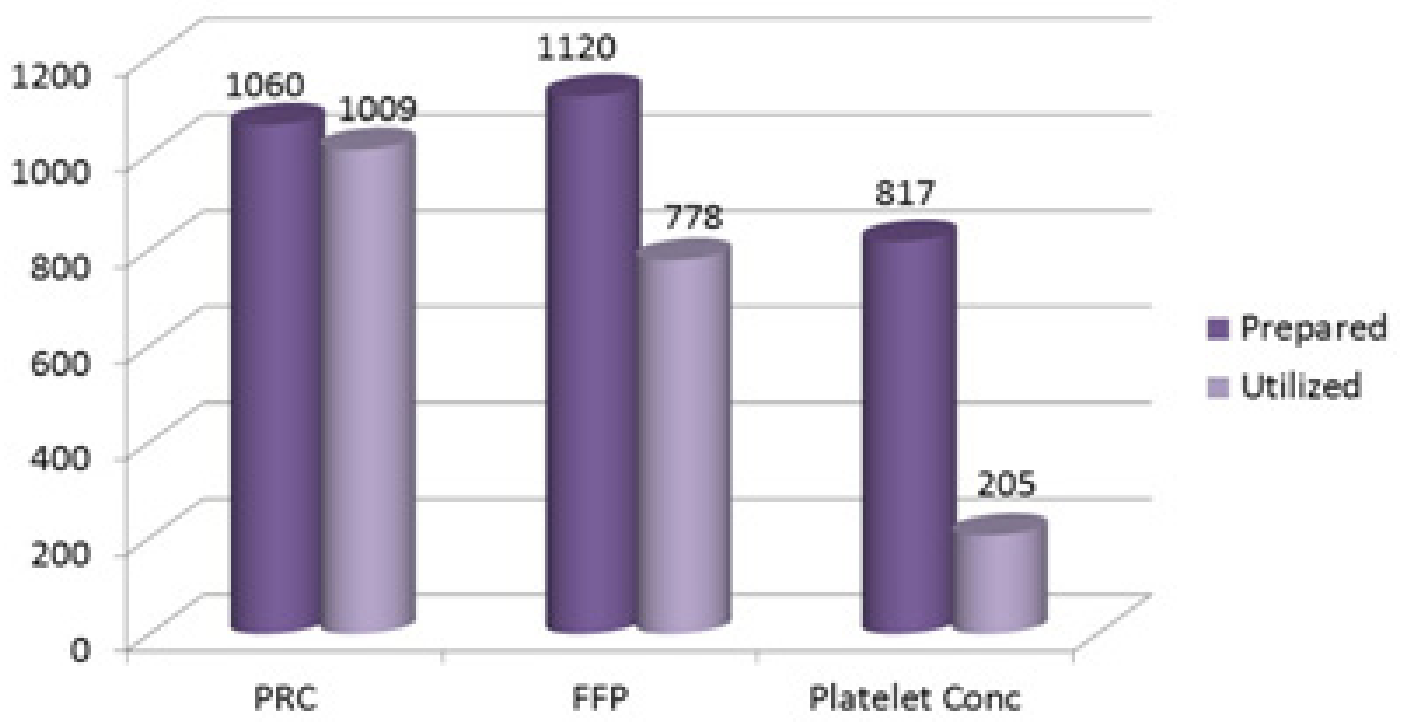

Fig. 2: Components prepared and utilized in one year (From 1st August 2016 to 31st July 2017) (PRC- Packed Red Cells, FFP- Fresh Frozen Plasma). 
The present study was done to assess the blood bank services of our hospital and improve the services by taking necessary measures. In our study many were male donors as females donors were deferred due to various reasons like low hemoglobin, menstrual periods etc. There was no maintenance of record for donors who were deferred on the basis of clinical history and limited physical examination. To eliminate this problem Blood bank has now prepared a proper deferral book and deferral entries are being done in it without fail.

Good number of collection was done in various blood donation camps arranged by social workers. Numbers of camp are increasing every year. Awareness about blood donation was created by personal communications and talks on need of blood donation and to clear the myths about blood donation. More efforts should be given to attract voluntary blood donors to meet the increased demand of blood and its components.

Standard Operating Procedures (SOPs) are maintained by blood bank to follow all the procedures accordingly. Revisions of these SOPs have been done depending on requirement and troubleshooting which had occurred during procedures annually. Also, regular internal and external training of the blood bank staff has been done.

Total number of blood issued in 2016 was 5897. Out of which majority of blood issues were to surgical wards compared to non-surgical wards as expected. We observed issues during night time were inappropriately issued without having any emergency indication. Sickle-cell anemia \& thalassaemia patients and patients having Below Poverty Line card (BPL) receive blood free of charge on their identity card.

Cross match to transfusion ratio (C: $\mathrm{T}$ ratio) is a ratio of number of blood units cross matched to the number of blood units issued. The ideal cross match to transfusion ratio(C: $\mathrm{T}$ ratio) should be 1:1.In our study the $\mathrm{C}$ : $\mathrm{T}$ ratio for year 2016 was 1.2:1 which is slightly higher. Higher C: $\mathrm{T}$ ratio can lead to increase workload on blood bank staff also wastage of blood bank reagents.

The screening of all donated blood for transfusiontransmitted infections(TTI), using the most appropriate and effective assays is done to test for hepatitis B and C, HIV $1 \& 2$ viruses, malaria and syphilis. Our present study showed among these diseases most were positive for hepatitis B followed by HIV. The TTI positivity in our study is high $(3.3 \%)$ so emphasis is now being given on proper screening of donors and promotion of voluntary donation.
Transfusion reactions occurred in few cases. Most common clinical symptoms were fever, vomiting, itching with rash. After complete investigations it was observed that all the blood units transfused were compatible and the reactions were probably allergic in nature.

Components were issued appropriately according to indications, but platelet concentrate were wasted more as its shelf life is only 7 days. In most developed countries, $75-100 \%$ of blood is transfused as components whereas, in developing countries, most blood transfused is as whole blood. In India, only $20 \%$ of blood units have been segregated into components. ${ }^{[11]}$ Awareness regarding component usage will ensure availability of components to needy patients and save many patients from transfusion reactions also.

Quality control is essential for continuous monitoring of equipment, competence of personnel and documentation $\&$ record maintenance. It is mandatory requirement to show digital display of temperature, thermograph, and alarm system in any storage equipment in blood bank. ${ }^{[12]}$ The records are maintained regarding calibrations of all instruments in blood bank. Also sterility testing has been done for empty CPDA bags as well as filled bags regularly by sending samples to microbiology section. For sustained improvement in practice, prospective monitoring must be continued indefinitely. ${ }^{[13,14]}$

Our results showed blood issues and donors are increasing day by day, so the workload. As we have less manpower it is overburdening our present staff.

\section{Conclusion}

A more comprehensive prospective audit is required to understand whether the transfusion services are being appropriately used for indications of blood use. Results obtained from these types of retrospective studies will provide opportunity for finding strategies in improving the blood bank services. We think our study pinpointed cardinal aspects of blood banking and rational use of blood and blood components which is useful for preparing future policies. The results of this type of study may be transferable to blood bank activities in other health enterprises.

\section{Reference}

1. Saran RK. Transfusion medicine technical manual. 2nd ed. Directorate General of Health Services, Ministry of health and family welfare, Government of India, New Delhi, India: WHO; 2003.

2. Arewa OP. Improving supply of safe blood and reducing the cost of transfusion service through heamovigilance. Niger Postgrad Med J. 2009;16:236-8. 
3. Benjamin RJ. Quality assurance, control \& improvement \& accreditation. In: Hillyer CD, Silberstein LE, Ness PM, Anderson KC, Roback JD, editors. Blood banking and transfusion medicine. 2nd ed. Philadelphia:Elsevier;2007. 230-7.

4. Kumar A, Sharma S, Ingole N, et al. An audit of blood bank services. J Edu Health Promot. 2014;3:59-61.

5. Ramani KV, Mavalankar DV, Govil D. Study of Bloodtransfusion Services in Maharashtra and Gujarat States, India. J Health PopulNutr. 2009;27:259-70.

6. Pinkerton PH. Blood bank audit. J ClinPathol. 1995;48:28386.

7. World Health Organization. Global data baon blood safetyreport 2001-02. Geneva: World Health Organization, 2004:5-17. (WHO/EHT/04.09).

8. Maccullogh J. Clinical uses of blood components. Transfusion medicine. 3rd ed. UK:Wiley-Blackwell;2012. 238-87.
9. Joshi GP, Landers DF. Audit in Transfusion Practice. J EvalClinPract. 1998;4:141-6.

10. Mallett SV, Peachey TD, Sanehi O, et al. Reducing red blood cell transfusion in elective surgical patients: the role of audit and practice guidelines. Anaesthesia. 2000;55:1013-19.

11. National AIDS Control Organization. An action plan for blood safety. New Delhi: Ministry of Health and Family Welfare, Government of India, 2003. 10 p.

12. Pilli G. Blood banking and transfusion medicine. Blood bank organization and its management. 1st ed. Belgaum: KLE university; 2012. p. 120-30.

13. Silver H, Tahhan HR, Anderson J, Lachman M. A noncomputerdependentprospective review of blood and blood component utilization. Transfusion. 1992;32: 260-65.

14. Marconi M, Almini D, Pizzi MN, RiccardiD, Bergamaschi $\mathrm{W}$, et al. Qualityassurance of clinical transfusion practice by implementation of the privilegeof blood prescription and computerized prospective audit of blood requests. Transfus Med. 1996;6:11-19.

*Corresponding author:

Dr. Shubhangi Narayan Jibhkate (Bawankule), E/66 New Nandanvan Colony, Nagpur 440023 Maharashtra, India.

Phone: +91 9373447439

Email: hubhanish@yahoo.com

Financial or other Competing Interests: None. 